\title{
LUPUS ERITEMATOSO SISTÉMICO JUVENIL: CARACTERÍSTICAS CLÍNICAS E INMUNOLÓGICAS EN UNA COHORTE ECUATORIANA DE 71 PACIENTES, EXPERIENCIA DE UN CENTRO
}

\author{
JUVENILE SYSTEMIC LUPUS ERYTHEMATOSUS: CLINICAL AND IMMUNOLOGICAL \\ CHARACTERISTICS IN AN ECUADORIAN COHORT OF 71 PATIENTS, \\ SINGLE-CENTER EXPERIENCE \\ LUUPUS ERITEMATOSO SISTÊMICO JUVENIL: CARACTERIISTICAS CLIINICAS EIMUNOLOOGICAS \\ EM UMA COORTE EQUATORIANA DE 71 PACIENTES, EXPERIÊNCIA DE UM ÚNICO CENTRO
}

\author{
CRISTINA HERRERA MORA', MÓNICA BOHORQUEZ VELASCO', JAVIER TOMALÁ HAZ
}

${ }^{1}$ Hospital Roberto Gilbert Elizalde, Guayaquil, Ecuador

RESUMEN

Introducción: el lupus eritematoso sistémico (LES) es una enfermedad autoinmune e inflamatoria multisistémica que afecta a todas las edades, el 20\% de los casos comienzan en la infancia y adolescencia. Las manifestaciones clínicas van desde lesiones cutáneas, hasta compromiso renal, hematológico o neurológico. El curso clínico se caracteriza por brotes y remisiones, y el pronóstico es impredecible. Objetivo: describir las características clínicas e inmunológicas de los pacientes con LES atendidos en el Roberto Gilbert E. Metodología: se trata de un estudio monocéntrico y retrospectivo. Se revisaron 75 historias clínicas electrónicas de la base de datos del hospital Roberto Gilbert Elizalde, de julio 2014 a enero 2018. Se recolectaron datos clínicos, de laboratorio, y el SLEDAI 2K (Systemic Lupus Erytematous Disease Activity Index 2K) (inicial y final). Los datos se analizaron con STATA 12.0. Las variables categóricas fueron analizadas usando Chi cuadrado. Resultados: se analizaron 71 historias clínicas, se excluyeron 4, por tener historia incompleta o completar criterios para síndrome de superposición; las restantes completaron criterios SLICC 2012. Las manifestaciones clínicas más frecuentes fueron: cutáneas (66,2\%), artritis $(43,6 \%)$, compromiso renal $(47,85)$, serositis $(35 \%)$ y alopecia $(32 \%)$. Los casos de LES familiar representaron el 11,9\%. Conclusiones: el sexo femenino, el compromiso renal y la hipoalbuminemia se encontraron asociadas a SLEDAI alto inicial. Además el compromiso renal se asoció a SLEDAl alto final, y a mortalidad. A los 4 años la supervivencia fue de 95,7\%. La remisión completa se logró en $28 \%$ de pacientes, y un $5 \%$ presentaba actividad grave.

PALABRAS CLAVE: lupus eritematoso sistémico, niños, hipoalbuminemia, sledai, proteinuria, mortalidad.

ABSTRACT

Introduction: systemic lupus erythematosus (SLE) is a multisystemic autoimmune and inflammatory disease that affects all ages, $20 \%$ of cases begin in childhood and adolescence. The clinical manifestations range are from skin lesions to renal, hematological, or neurological involvement. The clinical course is characterized by flare-ups and remissions, and the prognosis is unpredictable. Objective: describe the clinical and immunological characteristics of the patients with SLE treated at the Roberto Gilbert E. Methodology: this is a retrospective, monocentric study. 75 electronic medical records were reviewed, from the Roberto Gilbert Elizalde Hospital database, from July 2014 to January 2018. Clinical, laboratory data, and the SLEDAI 2K (Systemic Lupus Erythematosus Disease Activity Index 2K) (initial and final) were collected. The data were analyzed with STATA 12.0. The categorical variables were analyzed using Chi-square. Results: 71 medical records were analyzed, 4 were excluded due to having an incomplete history or completing criteria for overlap syndrome; the remaining ones completed the 2012 SLICC criteria. The most frequent clinical manifestations were cutaneous (66.2\%), arthritis (43.6\%), kidney involvement (47.85), serositis (35\%), and alopecia (32\%). Familial SLE cases represented $11.9 \%$. Conclusions: female sex, renal compromise, and hypoalbuminemia were found associated with initial high SLEDAI. Besides, renal involvement was associated with high-end SLEDAI and mortality. A 4 years survival was $95.7 \%$. Complete remission was achieved in $28 \%$ of patients, and $5 \%$ had severe activity.

KEYWORDS: lupus erythematosus sistemic, child, hypoalbuminemia, SLEDAL, proteinuria mortality.

RESUMO

Introdução: 0 lúpus eritematoso sistêmico (LES) é uma doença autoimune e inflamatória multissistêmica que afeta todas as idades, 20\% dos casos começam na infância e na adolescência. As manifestações clínicas variam desde lesões cutâneas até comprometimento renal, hematológico ou neurológico. 0 curso clínico é caracterizado por surtos e remissões, e o prognóstico é imprevisível. Objetivo: descrever as características clínicas e imunológicas dos pacientes com LES atendidos no hospital Roberto Gilbert E. Metodologia: este é um estudo retrospectivo e unicêntrico. Foram analisados 75 prontuários eletrônicos do banco de dados do Hospital Roberto Gilbert Elizalde, de julho de 2014 a janeiro de 2018. Foram coletados dados clínicos, laboratoriais e o SLEDAI 2K (Systemic Lupus Erytematous Disease Activity Index 2K) (inicial e final). Os dados foram analisados com o STATA 12.0. As variáveis categóricas foram analisadas pelo Qui-quadrado. Resultados: foram analisados 71 prontuários, 4 foram excluídos por apresentarem história incompleta ou preenchimento de critérios para síndrome de sobreposição; os demais preencheram os critérios do SLICC 2012. As manifestações clínicas mais frequentes foram: pele (66,2\%), artrite (43,6\%), envolvimento renal (47,85), serosite (35\%) e alopecia (32\%). Os casos familiares de LES representaram $11,9 \%$. Conclusões: sexo feminino, comprometimento renal e hipoalbuminemia foram encontrados associados ao SLEDAl inicial elevado. Além disso, 0 envolvimento renal foi associado a SLEDAl de alto nível e mortalidade. Aos 4 anos, a sobrevida foi de 95,7\%. A remissão completa foi alcançada em $28 \%$ dos pacientes e $5 \%$ apresentavam atividade grave.

PALAVRAS-CHAVE: lupus eritematoso, sistémico, infantis, hipoalbuminemia, SLEDAL, proteinuria, mortalidade. 
INTRODUCCIÓN

El lupus eritematoso sistémico es una enfermedad autoinmune e inflamatoria multisistémica que afecta todos los grupos etáreos, es más común en adultos y solo el $20 \%$ de los casos comienzan en la infancia y adolescencia. La mayor parte de los pacientes en pediatría debutan en la adolescencia con un franco predominio en el sexo femenino $(10: 1){ }^{1}$ Su forma de presentación es muy variable y para su identificación precoz se requiere un alto índice de sospecha; las manifestaciones clínicas van desde lesiones cutáneas, úlceras orales, hasta compromiso renal, hematológico o neurológico. El curso clínico se caracteriza por brotes y remisiones y el pronóstico es impredecible. ${ }^{1}$

La mayor parte del conocimiento de la enfermedad se basa en la experiencia en adultos, existen pocos datos epidemiológicos del lupus juvenil ${ }^{(2,3)}$

METODOLOGÍA

Estudio de cohortes, mono-céntrico, retrospectivo, el periodo de enrolamiento de los pacientes fue desde julio 2014 hasta enero 2018. Se revisaron 75 historias clínicas electrónicas de la base de datos del hospital Roberto Gilbert E. Se excluyeron 4 historias clínicas, una por incompleta, y 3 por completar criterios para síndrome de superposición. Todas las historias clínicas restantes completaron criterios SLICC 2012 con una edad de inicio antes de los 18 años.

Las variables clínicas y de laboratorio, así como los parámetros de actividad se definieron en conjunto por 2 reumatólogos pediátricos, y 1 nefrólogo pediátrico. Los datos se recolectaron de febrero a marzo 2018.

Los datos demográficos incluían edad de inicio, sexo, antecedentes familiares de autoinmunidad y raza, que fue clasificada en: mestiza, afroecuatoriana, blanca, asiática o con ascendencia asiática, e indígena. Dentro de las manifestaciones clínicas incluyeron las cutáneas agudas (rash malar, ampolloso, necrólisis epidérmica tóxica, rash fotosensible), cutáneo crónico, aftas orales, alopecia, compromiso articular, Sjogrën secundario, compromiso pulmonar (con datos clínicos, y TAC-AR pulmonar compatible), anemia hemolítica autoinmune, poliserositis, compromiso renal (con proteinuria y/o hematuria) y compromiso neurológico (convulsiones, psicosis, corea). Se revisaron los datos de laboratorio del debut: hemograma completo, PCR, VSG, TGO, TCP, albúmina, globulina, complemento, orina general, proteinuria en orina de 24 horas e índice proteína/creatinina en orina parcial. La técnica para obtener los ANA y el anti DNA fue por ELISA o por inmunofluorescencia indirecta. Los anti ENA (anti Smith, anti RNP, anti Ro, anti La) fue evaluado por ELISA o por LIA (inmunoensayo lineal). Los anticuerpos antifosfolipídicos al ser un examen que se realiza fuera del hospital, no se pudieron realizar en todos los pacientes.

El índice de Actividad lúpica usado fue el SLEDAI2K (Systemic Lupus Erythematosus Disease Activity Index 2000). Para evaluar la respuesta al tratamiento se analizó el SLEDAI al debut y en la fecha de última consulta. Los resultados se expresaron en 4 variables: remisión/actividad mínima (SLEDAI 0-2), actividad leve-moderada (SLEDAI más 3), actividad alta (SLEDAI 12 o más) y muerte. Los datos se analizaron con STATA 12.0. Las variables continuas fueron expresadas como media +/- desviación estándar, y las categóricas en porcentajes. Las variables categóricas fueron analizadas usando Chi cuadrado.

RESULTADOS

En la presente cohorte la distribución por sexo fue masculino $14.08 \%$ (10 pacientes) y femenino $85,92 \%$ (61 pacientes). La edad de presentación fue de 4 a 17 años, con una media de 12,19 y una desviación estándar de 2,98. De los pacientes, el $11,27 \%$ (8 pacientes) tenían antecedentes de familiares con lupus y el 15\% (11 pacientes) tenían autoinmunidad en familiares de primer o segundo grado. La mayoría eran de raza mestiza 95,7\% (68), $2,82 \%$ blancos, y $1,4 \%$ negros. Todos completaban los criterios SLICC 2012; y el 81,69\%, los criterios ACR 1997.

\section{TABLA 1. DATOS EPIDEMIOLÓGICOS DE LOS PACIENTES}

\begin{tabular}{lr}
\hline PARÁMETROS & VALOR \\
\hline Número total de pacientes & 71 \\
\hline Edad (Media, Desviación stándar) & $12.19( \pm 2.988)$ \\
\hline Días al diagnóstico (Media, Desviación stándar) & $95.95( \pm 118.17)$ \\
\hline $\begin{array}{l}\text { Sexo } \\
\text { Radio Femenino/masculino }\end{array}$ & Femenino 61 Masculino 10 \\
Porcentaje & Masculino: $14,08 \%$ \\
\hline Antecedentes patológicos personales (valor & Femenino: $85,92 \%$ \\
absoluto/ porcentual) & $8(11,27 \%)$ \\
\hline Raza (valor absoluto/ porcentual) & Mestizos $68(95.77 \%)$ \\
Criterios ACR (valor absoluto/ porcentual) & Negro $1(1.41 \%)$ \\
Criterios SLICC (valor absoluto/ porcentual) & Blanco $2(2.82 \%)$ \\
\hline Meses seguimiento (valor absoluto/ porcentual) & $58(81.69 \%)$ \\
\hline Meses de enfermedad (valor absoluto/ porcentual) & $71(100 \%)$ \\
\hline
\end{tabular}


Dentro de las manifestaciones clínicas encontradas, las más frecuentes fueron: cutáneas $66,2 \%$, renal $47,8 \%$, artritis $43,66 \%$, alopecia $32,3 \%$, serositis 35,2\%, AHAI (anemia hemolítica autoinmune) $21,4 \%$, aftas orales $18,3 \%$, compromiso neurológico $12 \%$. Dentro de las manifestaciones cutáneas, hubo 1 paciente con lupus ampollar y uno con Steven Johnson (Síndrome de Rowell). Dentro de las manifestaciones infrecuentes, un paciente tuvo déficit transitorio de factor VIII como manifestación inicial del lupus, y una paciente tenía Síndrome de Mc Cune Albrigth como comorbilidad.

\section{TABLA 2. MANIFESTACIONES CLÍNICAS DE LOS PACIENTES}

\begin{tabular}{|lr}
\hline MANIFESTACIÓN CLÍNICA & $\begin{array}{r}\text { FRECUENCIA (VALOR } \\
\text { ABSOLUTO/ PORCENTUAL) }\end{array}$ \\
\hline Cutáneo & $47(66,2 \%)$ \\
\hline Ampollar & $1(0,71 \%)$ \\
\hline Aftas orales & $13(18,3 \%)$ \\
\hline Alopecia & $23(32,3 \%)$ \\
\hline Artritis & $31(43,66)$ \\
\hline Sjögren & $2(2,8 \%)$ \\
\hline Compromiso pulmonar & $4(5,71 \%)$ \\
\hline Serositis & $25(35,2 \%)$ \\
Renal & $34(47,8 \%)$ \\
\hline Neurológico & $9(12 \%)$ \\
AHAl & $15(21,4 \%)$ \\
\hline Tiroiditis & $3(2,13 \%)$ \\
\hline Parotiditis & $3(2,13)$ \\
\hline Epiescleritis & $1(0,71 \%)$ \\
Vitiligo & $1(0,71 \%)$ \\
Déicit transitorio de factor VIII & $1(0,71 \%)$ \\
\hline Steven Johnsons (Rowell) & $1(0,71 \%)$
\end{tabular}

En el laboratorio inicial se encontró que el 7,04\% de los pacientes tuvieron cilindruria, 39,44\% hematuria, de los cuales el 8,45\% era hematuria pura; el 39,44 tuvo proteinuria y 8,45\% proteinuria pura. El 57,7\% de pacientes tuvieron anemia, $21,4 \%$ fue hemolítica autoinmune, y el $28,17 \%$ tuvieron coombs positivo. El $30 \%$ de los pacientes tuvieron plaquetopenia, $84 \%$ de tuvo hipocomplementemia. El ANA fue positivo en el 83,1\% de los pacientes y los anti ENA en el 93\%. El valor promedio de PCR fue 3,2 (valor referencial 0-3) y la eritrosedimentación 32 (valor referencial 0-25 $\mathrm{mm} / \mathrm{h}$ ). Los anticuerpos antifosfolipídicos fueron positivos en el 11,27\% de pacientes, negativos en $61.97 \%$ y no se pudieron realizar en el $26,27 \%$ de los pacientes, (Tabla 3). Desafortunadamente del total de 30 pacientes con compromiso renal, solo a 10 pacientes se les realizó biopsia renal, de ellos 5 tenían Glomerulonefritis clase IIB (50\% de las biopsias), 3 pacientes clase III (30\%), y 2 pacientes estadio IV (20\%). Al resto de pacientes no se les pudo realizar la biopsia renal por motivos económicos o por falta de consentimiento de los padres.
En el laboratorio inicial se encontró que el 7,04\% de los pacientes tuvieron cilindruria, 39,44\% hematuria, de los cuales el 8,45\% era h maturia pura; el 39,44 tuvo proteinuria y $8,45 \%$ proteinuria pura. El $57,7 \%$ de pacientes tuvieron anemia, $21,4 \%$ fue hemolítica autoinmune, y el $28,17 \%$ tuvieron coombs positivo. El 30\% de los pacientes tuvieron plaquetopenia, $84 \%$ de tuvo hipocomplementemia. El ANA fue positivo en el $83,1 \%$ de los pacientes y los anti ENA en el 93\%. El valor promedio de PCR fue 3,2 (valor referencial 0-3) y la eritrosedimentación 32 (valor referencial 0-25 mm/h). Los anticuerpos antifosfolipídicos fueron positivos en el 11,27\% de pacientes, negativos en $61.97 \%$ y no se pudieron realizar en el $26,27 \%$ de los pacientes.

\section{TABLA 3. DATOS DE LABORATORIO DE LOS PACIENTES EN SU VALORACIÓN INICIAL}

\begin{tabular}{|c|c|}
\hline PARÁMETRO & VALOR \\
\hline Porcentaje de pacientes con cilindruria & $7.04 \%$ \\
\hline Porcentaje de pacientes con hematuria & $\begin{array}{r}39.44 \% \\
\text { Solo hematuria } 8,45 \%\end{array}$ \\
\hline Porcentaje de pacientes con proteinuria & $\begin{array}{r}39.44 \% \\
\text { Solo proteinuria } 8.45 \%\end{array}$ \\
\hline $\begin{array}{l}\text { Proteinuria } \mathrm{mg} / \mathrm{m} 2 / \mathrm{h} \text { (media/desviación } \\
\text { estándar }\end{array}$ & $77.29( \pm 56.08)$ \\
\hline $\begin{array}{l}\text { Índice proteína/creatinina en orina parcial } \\
\text { (media/desviación estándar) }\end{array}$ & $11.28( \pm 36.66)$ \\
\hline Albumina g/dL (media/desviación estándar) & $3.24( \pm 1.16)$ \\
\hline Globulina g/dL (media/desviación estándar) & $3.74( \pm 1.04)$ \\
\hline TGO UI/L (media/desviación estándar) & $109.59( \pm 230.62)$ \\
\hline TGP UI/L (media/desviación estándar) & $81.78( \pm 181.08)$ \\
\hline Anemia (absoluto/porcentual) & $41(57.75 \%)$ \\
\hline Coombs positivo (absoluto/porcentual) & $20(28.17 \%)$ \\
\hline Hb (media/desviación estándar) & $9.56( \pm 2.83)$ \\
\hline Leucopenia (absoluto/porcentual) & $15(21.13 \%)$ \\
\hline Leucocitos (media/desviación estándar) & $7,171.26( \pm 3986.04)$ \\
\hline Linfopenia (absoluto/porcentual) & $12(17.14 \%)$ \\
\hline Linfocitos (media/desviación estándar) & $1,878.54( \pm 1059.83)$ \\
\hline Plaquetopenia (absoluto/porcentual) & $22(30.99 \%)$ \\
\hline Plaquetas (media/desviación estándar) & $227,957.7( \pm 11,7136.8)$ \\
\hline Hipocomplementemia (absoluto/porcentual) & $60(84.51 \%)$ \\
\hline C3 (media/desviación estándar) & $56.63( \pm 31.78)$ \\
\hline C4 (media/desviación estándar) & $7.64( \pm 6.03)$ \\
\hline PCR (media/desviación estándar) & $3.20( \pm 6.72)$ \\
\hline VSG (media/desviación estándar) & $32.42( \pm 20.33)$ \\
\hline ANA (absoluto/porcentual) & $\begin{array}{r}\text { Positivo } 59(83.10 \%) \\
\text { Negativo } 5(7.04 \%) \\
\text { No realizado } 7(9.86 \%)\end{array}$ \\
\hline Anti ENA (absoluto/porcentual) & $\begin{array}{r}\text { Positivo } 63(90 \%) \\
\text { Negativo } 7(10 \%)\end{array}$ \\
\hline Anti Smith (absoluto/porcentual) & $51(71.83 \%)$ \\
\hline Anti DNA (absoluto/porcentual) & $52(73.24 \%)$ \\
\hline Antifosfolipidicos (absoluto/porcentual) & $\begin{array}{r}\text { Negativos } 44(61.97 \%) \\
\text { Positivos } 8(11.27 \%) \\
\text { Desconocido } 19(26.76 \%)\end{array}$ \\
\hline
\end{tabular}


Respecto al tratamiento: El 100\% de los pacientes recibió hidroxicloroquina como inmunomodulador, $52 \%$ recibió azatioprina, $45 \%$ micofenolato de mofetilo, $25 \%$ ciclofosfamida, $12,6 \%$ metotrexate, $9,8 \%$ Inmunoglobulina endovenosa, y $7 \%$ rituximab. Cabe señalar que el $56,3 \%$ de pacientes (40) recibieron 1 inmunosupresor, 33,8\% ${ }^{(24)}$ pasaron por 2 inmunosupresores, 5,6\% ${ }^{(4)}$ por 3 inmunosupresores y 2,8\% (3) por 4 inmunosupresores, sólo en 1 paciente con nefritis lúpica se necesitó un protocolo de doble inmunosupresión (rituximabciclofosfamida en los días 0-1, 14-15) (Lehman et al. 2014)1por haber sido refractaria previamente a micofenolato de mofetilo, ciclofosfamida mensual, rituximab 4 dosis, y ciclofosfamida bimensual.

TABLA 4. TRATAMIENTO INMUNOSUPRESOR USADO EN LOS PACIENTES
DURANTE TODO SU SEGUIMIENTO
\begin{tabular}{|lr}
\hline INMUNOSUPRESOR & PORCENTAJE/ N \\
\hline Hidroxicloroquina & $100 \%(71)$ \\
\hline Azatioprina & $52 \%(37)$ \\
\hline Micofenolato de Mofetilo & $45 \%(32)$ \\
Ciclofosfamida & $25 \%(18)$ \\
Metotrexate & $12,6 \%(9)$ \\
\hline Rituximab & $7 \%(5)$ \\
\hline Inmunoglobulina endovenosa & $9,8 \%(7)$
\end{tabular}

De los pacientes el 53,52\% estaban en remisión completa o actividad mínima de la enfermedad (SLEDAI 0-2) en la última cita, el 46,48\% estaba en actividad continua, y de ellos el 5,63\% tenía actividad grave. El SLEDAI inicial promedio fue de 10,59 , y el SLEDAI promedio en la última visita fue de 3,51. El número de reactivaciones fue de 1,61 $+/-0,83$. El número de meses de enfermedad de los pacientes fue de 29 meses en promedio, con un promedio de seguimiento en nuestra institución de 18 meses. El porcentaje de mortalidad a los 4 años fue $4,23 \%$ (3 pacientes), de los 34 pacientes con compromiso renal 3 fallecieron, es decir el 8,8\% .

tABLA 5. RESPUESTA DE LOS PACIENTES AL tRATAMIENTO INMUNOSUPRESOR

\begin{tabular}{|c|c|}
\hline ACTIVIDAD LÚPICA & VALOR \\
\hline Remisión completa ( N/porcentaje) & $20(28,17 \%)$ \\
\hline Actividad mínima (N/porcentaje) & $18(25.35 \%)$ \\
\hline Actividad continua (N/porcentaje) & $\begin{array}{r}33(46,48 \%) \text {, leve- moderada } 29 \\
(40,85 \%), \text { Grave } 4(5,63 \%)\end{array}$ \\
\hline Numero de brotes (N/porcentaje) & $\begin{array}{r}1=40(56.34 \%) ; 2=21 \\
(29.58 \%) ; 3=7(9.86 \%) ; 4=3 \\
(4.23 \%)\end{array}$ \\
\hline $\begin{array}{l}\text { Dosis de corticoide en última consulta } \\
\text { (mg/Kg/día) (media/ desviación estandar }\end{array}$ & $0.34( \pm 0.32)$ \\
\hline $\begin{array}{l}\text { SLEDAl inicial (media /desviación } \\
\text { estándar) }\end{array}$ & $10.59( \pm 4.50)$ \\
\hline $\begin{array}{l}\text { SLEDAl en última consulta (media/ } \\
\text { desviación estandar). }\end{array}$ & $3.51( \pm 3.89)$ \\
\hline $\begin{array}{l}\text { Numero de Reactivaciones (media/ } \\
\text { desviación estándar) }\end{array}$ & $1.61( \pm 0.83)$ \\
\hline
\end{tabular}

Se analizaron las variables categóricas con el test Chi cuadrado, y se encontró que el sexo femenino estaba asociado con actividad lúpica alta al inicio de la enfermedad con p significativa, así mismo el compromiso renal y la hipoalbuminemia. El compromiso renal se asoció a riesgo de mortalidad con una p 0,065.

\section{TABLA 6. ANÁLISIS ESTADÍSTICO BIVARIADO DE LAS CARACTERISTIICAS CLÍNICO EPIDEMIOLÓGICAS Y SU RELACIÓN CON LA ACTIVIDAD DE LA ENFERMEDAD.}

\begin{tabular}{lr}
\hline ASOCIACIONES ENTRE: & P \\
\hline Sexo femenino \# actividad lúpica alta & 0.015 \\
APF \# actividad lúpica alta & 0.369 \\
\hline Anti Smith \# actividad lúpica alta inicial & 0.876 \\
\hline ANA \# actividad lúpica alta inicial & 0.534 \\
\hline Anti ENA \# actividad lúpica alta inicial & 0.347 \\
\hline Albumina \# actividad lúpica alta inicial & 0.0001 \\
\hline Cutáneo agudo \# actividad baja inicial & 0.0380 \\
Renal \# actividad alta inicial & 0.0001 \\
\hline Renal \# actividad alta final & 0.033 \\
\hline AHAl \# actividad alta inicial & 0.23 \\
\hline AHAl \# actividad alta final & 0.85 \\
Neurológico \# actividad alta inicial & 0.192 \\
Neurológico \# actividad alta final & 0.436 \\
Hipocomplementemia \# hematuria & 0.3726 \\
\hline Hipocomplementemia \# proteinuria & 0.1192 \\
AHAl \#mortalidad & 0.1192 \\
Neurológico \#mortalidad & 0.5031 \\
\hline Renal \#mortalidad & 0.065 \\
APF = Antecedentes patológicos familiares. ANA=Anticuerpos antinucleares. Anti \\
ENA=Anti Antigenos extraíbles del núcleo. AHAl =Anemia hemolítica autoinmune.
\end{tabular}

\section{DISCUSIÓN}

La incidencia anual del LESj reportada en otros países como USA es de 10 por cada 100,00 niños(Hiraki et al. 2012). ${ }^{4}$ En nuestro país aún no se han realizado estudios epidemiológicos que permitan determinar su incidencia y prevalencia. En el repositorio de la Universidad Católica Santiago de Guayaquil se publicó una cohorte ecuatoriana de pacientes adultos con LES, ${ }^{6}$ pero ninguna juvenil. El presente estudio es retrospectivo, monocéntrico y la n es pequeña; pese a esas limitaciones, representa la realidad de los pacientes con LESj que habitan en la costa ecuatoriana, he ahí la relevancia del estudio.

El punto de corte respecto a la edad en el LESj no está establecido, en las cohortes publicadas difiere según los países (antes de los 14, 16 o 18 años), el valor de corte de este estudio fue de 18 años (edad máxima de atención pediátrica en nuestro hospital). La edad media al diagnóstico al igual que 
en otras series fue de 12 años. ${ }^{2,6-8}$ Solo $2,8 \%$ de los pacientes tenían menos de 6 años igual que en otras cohortes, excepto en la cohorte brasileña que tuvo $4.6 \%$ pero el estudio era multicéntrico con una $\mathrm{N}$ de 847. Como en otras series el porcentaje de mujeres afectadas es alto $85 \%$ y la relación mujer/ hombre es de $6,1: 1,4,7,8$.

Las manifestaciones clínicas más frecuentes en este estudio fueron: cutáneas $(66,2 \%)$, artritis $(43,6 \%)$, compromiso renal $(47,85)$, serositis (35\%), alopecia $(32 \%)$ y AHAI $(21 \%)$. Otras cohortes de LESj reportaron entre 21 al 46\% de pacientes con compromiso del SNC y un 46-65\% de pacientes con nefritis lúpica. La serositis y la alopecia se encontraron en igual porcentaje que en otras series. Llama la atención que en esta cohorte el número de pacientes con compromiso neurológico no fue elevado (12\%).

Por otro lado, el lupus discoide es muy raro en niños, en la presente casuística ningún paciente lo presentó. La mayoría de cohortes reportan pocos o ningún paciente con lupus discoide, salvo en la cohporte brasileña de Gomes y col. ${ }^{7-9} \mathrm{En}$ la cohorte ecuatoriana de pacientes adultos con LES reportaban porcentajes mucho más elevados de compromiso hematológico $(87 \%)$, renales $(76 \%)$, articular $(76 \%$ y neuropsiquiátrico $(33 \%)$, si bien el enfoque de esta cohorte era orientado a pacientes hospitalizados en área crítica. El compromiso cutaneomucoso fue similar que en este estudio $(61 \%) .5$

Por lo general los casos familiares de LES representan el $10 \%$. En este estudio fue muy similar, el $11,9 \%$ de pacientes tenían algún familiar de 1er o 2do grado (padres, hermanos, tíos, abuelos) con lupus eritematoso sistémico. El tener antecedentes familiares no se asoció con un SLEDAI alto, distinto a lo comparado por único estudio de Abdwani et al. ${ }^{11}$

Respecto al laboratorio, la incidencia de LES ANA negativo ha sido estimada en $1-5 \%$ de pacientes, en el presente reporte el $5 \%$ los de pacientes era ANA negativo y esto se debe a una falla en la técnica atrapamiento de ANA en complejos inmunes circulantes. ${ }^{10} \mathrm{La}$ albumina baja se encontró asociada a actividad lúpica alta, igual que ya se ha reportado en LES en adultos. ${ }^{12} \mathrm{El}$ compromiso renal se asoció con SLEDAI alto inicial y final, y también con mortalidad, con una p significativa, como también lo notaron en otras series.
Contrariamente a lo encontrado en otras cohortes, en la presente el compromiso neurológico no se asoció ni con SLEDAI alto, ni con mortalidad.6,7

El tratamiento de lupus eritematoso en niños está basado en series pediátricas son pequeñas ${ }^{3}$ o por la extrapolación de los estudios randomizados realizados en adultos. ${ }^{13,14}$ Estudios a gran escala en niños son difíciles por 2 razones, porque el lupus y más el lupus juvenil es un grupo minoritario y por falta de financiamiento. Durante el transcurso de los años la terapéutica ha cambiado y mejorado. ${ }^{18,19}$

En el 1960 las terapias eran limitadas y tóxicas y $50 \%$ de los niños morían en los 2 primeros años. Hoy en día existen más inmunosupresores que en el pasado, y los actuales han reducido la toxicidad, mejorando el pronóstico. Actualmente, la supervivencia a los 5 años es de un 95 a $97 \%$ y de los pacientes renales excede el $90 \% .{ }^{20}$ Si bien en el país no existen todas las terapias biológicas, el uso de micofenolato de mofetilo y de Rituximab ha mejorado los resultados en los pacientes con LESj refractarios a terapias convencionales como la azatioprina o la ciclofosfamida.

En este estudio la supervivencia a los 4 años fue de $95,7 \%$. La remisión completa se logró solo en un $28 \%$ de pacientes, menos que en otras cohortes; Cabral et al reportó 32\% de remisión en pacientes con LESj en un seguimiento de 5,5 $(+/-5,4)$ años. Pese a esto la mitad de los pacientes estaba en el grupo de actividad mínima/ remisión completa, y sólo un $5 \%$ presentaba actividad grave.

\section{CONCLUSIÓN Y RECOMENDACIONES}

Las manifestaciones clínicas más frecuentes fueron: cutáneas $(66,2 \%)$, artritis $(43,6 \%)$, compromiso renal $(47,85)$, serositis $(35 \%)$ y alopecia (32\%). La hipocomplementemia estuvo presente en el $84,5 \%$ de pacientes, el ANA positivo en $83,1 \%$ y los anti ENA en el $90 \%$. Los casos de LES familiar representaron el 11,9\%, el LES familiar no se asoció con SLEDAI alto inicial.

El sexo femenino, el compromiso renal y la hipoalbuminemia se encontraron asociadas a SLEDAI alto inicial. Además el compromiso renal se asoció a SLEDAI alto final, y a mortalidad. 
A los 4 años la supervivencia fue de 95,7\%. La remisión completa se logró en $28 \%$ de pacientes, la mitad de los pacientes estaba en el grupo de actividad mínima/remisión completa (53,52\%), $46,48 \%$ tenían actividad continua, y solo un $5 \%$ presentaba actividad grave.

Esta es la primera cohorte de Lupus eritematoso sistémico juvenil presentada en nuestro país, en el futuro se espera poder contar con estudios multicéntricos que permitan evaluar la situación global del país.

Posteriormente también se debería analizar el apego de los pacientes al tratamiento y la pérdida de pacientes durante el seguimiento y como estas 2 variables pueden influir en el pronóstico de la enfermedad.

\section{REFERENCIAS BIBLIOGRAFICAS}

1. Espada G., Maragón C. Manual práctico de reumatología pediátrica / lera edición, Buenos Aires: Nobuko, 2005. ISBN 987-584-032-7.

2. Font J, Cervera R, Espinosa G, Pallarés L, Ramos-Casals M, Jiménez S, García-Carrasco $M$, Seisdedos L, Ingelmo M. Systemic lupus erythematosus (SLE) in childhood: analysis of clinical and immunological findings in $34 \mathrm{pa}^{-}$ tients and comparison with SLE characteristics in adults. Ann Rheum Dis. 1998 Aug;57(8):456-9.

3. Lehman T, Singh C, Ramanathan A, et al. Prolonged improvement of chilhood onset systemic lupus erythematosus following systematic administration of rituximab and cyclophosphamide. Pediatric Rheumatology 2014, 12:3 http://www.ped-rheum.com/content $/ 12 / 1 / 3$

4. Hiraki LT, Feldman CH, Liu J, et al. Prevalence, incidence, and demographics of systemic lupus erythematosus and lupus nephritis from 2000 to 2004 among children in the US medicaid beneficiary population. Arthritis \& Rheumatism Vol. 64, No. 8, August 2012, pp 2669-2676.

5. García K, Mera G, Zuñiga A. Características clínicas del Lupus Eritematoso Sistémico en pacientes ingresados en el Hospital Luis Vernaza de Guayaquil, con énfasis en Terapia Intensiva, durante el período 2015-2017. repositorio.ucsg. edu.ec/bitstream/3317/9332/1/T-UCSG-PREMED-606.pdf

6. MacKie, F. E, Kainer, G. Adib, N. The national incidence and clinical picture of SLE in children in Australia. A report from the Australian Paediatric Surveillance Unit. Lupus (2015) 24, 66-73. http://lup.sagepub.com
7. Cabral M, Escobar C, Conde $M$ et al. Juvenile systemic lupus erythematosus in Portugal: Clinical and immunological patterns of disease expression in a cohort of 56 patients. acta reumatol port. 2013;38:274-285

8. Abdel-Hafez MA, Abdel-Nabi H. Juvenile systemic lupus erythematosus: onset patterns and short-term outcome in Egyptian children, a single-center experience. Lupus (2015) 0, 1-7 . http://lup.sagepub.com

9. Gomes RC, Silva M F, Kozu K et al. Features of 847 Childhood-Onset Systemic Lupus Erythematosus Patients in Three Age Groups at Diagnosis: A Brazilian Multicenter Study. Arthritis Care \& Research Vol. 68, No. 11, November 2016, pp 1736-1741

10. Caltik A, Demircin G, Bülbül M, et al. An unusual case of ANA negative systemic lupus erythematosus presented with vasculitis, longstanding serositis and full-house nephropathy. Rheumatol Int 2013, Vol 33, No. 1, 219-222

11. Abdwani, R, Hira, M.Juvenile systemic lupus erythematosus in the Sultanate of Oman: Clinical and immunological comparison between familial and non-familial cases. Lupus (2011) 20, 315-319 http://lup.sagepub.com

12. Yip Jonathan, Aghdassi Elaheh, Su Jiandong. Serum albumin as a marker for disease activity in patients with systemic lupus erythematosus. J Rheumatol 2010; 37:8

13. Petty R, Laxer R, Lindsley C, Wedderburn L. Textbook of pediatric rheumatology. 7 th edition. Systemic Lupus Erythematosus, Capítulo 23. Página 285-317

14. Ruiz-Irastorza G, Espinosa G, Frutos MA, Jiménez-Alonso J, Praga $M$ et al. Diagnosis and treatment of Lupus nephritis: Consensus document from the systemic auto-immune disease group (GEAS) of the Spanish society of internal medicine (SEMI) and the Spanish society of nephrology (S.E.N.) Nefrologia 2012;32(Suppl.1):1-35

15. Pons-Estel G, Alarcón, G, Mc Gwin, G, Danila $M$, Zhang J, et al. Protective Effect of Hydroxychloroquine on Renal Damage in Patients with Lupus Nephritis: Data from LUMINA, a Multiethnic U.S. Cohort. Arthritis Rheum. 2009 June 15; 61(6): 830-839.

16. Shinjo, S, Bonfá, E, Wojdyla D et al. Antimalarial treatment may have a time-dependent effect on lupus survival: Data from a multinational Latin American inception cohort. Arthritis \& rheumatism. Vol. 62, No. 3, March 2010, pp 855-862 
17. Groot N, De Graeff N, Marks S et al. European evidence-based recommendations for the diagnosis and treatment of childhood-onset lupus nephritis: The SHARE initiative. Ann Rheum Dis 2017;0:1-9. http:// dx. doi. org/ 10. 1136/ annrheumdis- 2017- 211898.

18. Groot N, De Graeff N, Marks S et al. European evidence-based recommendations for the diagnosis and treatment of childhood-onset systemic lupus erythematosus: The SHARE initiative. Ann Rheum Dis 2017;0:1-9. http:// dx. doi. org/ 10. 1136/ annrheumdis-2016-210960
19. Sag E., Tartaglione A., et al. Performance of the new SLICC classification criteria in childhood systemic lupus erythematosus: a multicentre study. Clinical and Experimental Rheumatology 2014; 32: 440-444.

20. Scott E., Wenderfer, et al. Advances in the Care of Children with Lupus Nephritis, Pediatric Research November 2016; doi:10.1038/pr.2016.247. 